International Journal of Biomedicine | June 2021 - Volume 11, Issue Suppl_1: Abstracts from the Third Russian International Conference "Cryo-electron microscopy 2021: achievements and prospects"

\author{
POSTER ABSTRACT PRESENTATIONS
}

SESSION TITLE: ADVANCES IN EM TECHNOLOGY AND PROCESSING

DOI: 10.21103/IJBM.11.Supp1_1.P6

\title{
Abstract P-6: Automated Pipeline for Parametrization of the Coarse- Grained Models for Biomolecular Simulations
}

\section{Philipp S. Orekhov}

Faculty of Biology, Lomonosov Moscow State University, Moscow, Russia

Background: Despite the advances in computational techniques and computing resources, the atomistic simulations are still limited in terms of attainable simulation time (up to $1-100 \mu \mathrm{s}$ ) and system size (up to millions of atoms). Various coarse-grained models provide a valuable alternative to the fully atomic simulations but they largely rely upon the rigorous parametrization procedure. The latter is required to obtain a set of coarse-grained force field parameters adequately reproducing the behavior of the original full-atom system.

Here, we report an automated pipeline designed for the parametrization of coarse-grained models of small organic compounds and polymers. While it is tailored to the MARTINI coarse-graining framework, it can also be applied to other force fields following a similar coarse-graining strategy.

Methods: Given the all-atom trajectory and the mapping (i.e., a table describing the correspondence between atoms and coarse-grained particles), the parametrization procedure iteratively optimizes bonded parameters of the coarse-grained model such that the bond, angle, (if required) dihedral histograms obtained from a series of coarse-grained simulations gradually converge to the target distributions derived from the full-atom trajectory mapped to the CG resolution.

Results: The developed pipeline accepts as an input a full-atom trajectory of a molecule to be parametrized as well as the mapping between the full-atom and coarse-grained models. Based on this input, the initial coarse-grained model is built using Boltzmann inversion, which is a subject for the following iterative optimization. At each iteration, the convergence of bonded terms is checked based on the overlap of respective histograms derived from full-atom and coarse-grained simulations and, in case of satisfactory convergence, the optimization is stopped. In addition to the parametrization of individual 
compounds, the pipeline also allows deriving models for polymers consisting of repetitive units.

The proposed here approach has been successfully used to create coarse-grained topologies for various small molecular weight compounds, such as drugs and antiseptics, as well as for detergents (DDM) and amphiphilic polymers (SMA, DIBMA) stabilizing lipodiscs - nanosized lipid particles widely exploited in structural studies of membrane proteins. The later models proved themselves particularly helpful for the interpretation of experimental data from small-angle scattering, EM, and EPR experiments

Conclusion: We expect that the developed pipeline would support the fast and straightforward development of coarse-grained models for simulations of biomolecular systems and new materials. Such simulations can be especially useful for the interpretation of experimental data obtained by, e.g., small-angle scattering and Cryo-EM experiments.

The pipeline is available online at https://github.com/porekhov/cgmktop.

Key words: molecular dynamics $\bullet$ coarse-grained models.

This work was supported by the Russian Science Foundation (Grant No. 1974-00065)

*Corresponding author: Philipp Orekhov.E-mail: orekhov@mail.bio.msu.ru

International Journal of Biomedicine. 2021;11 Suppl 1: S13.

doi: 10.21103/IJBM.11.Suppl_1.P6

(C)2021 International Medical Research and Development Corporation 